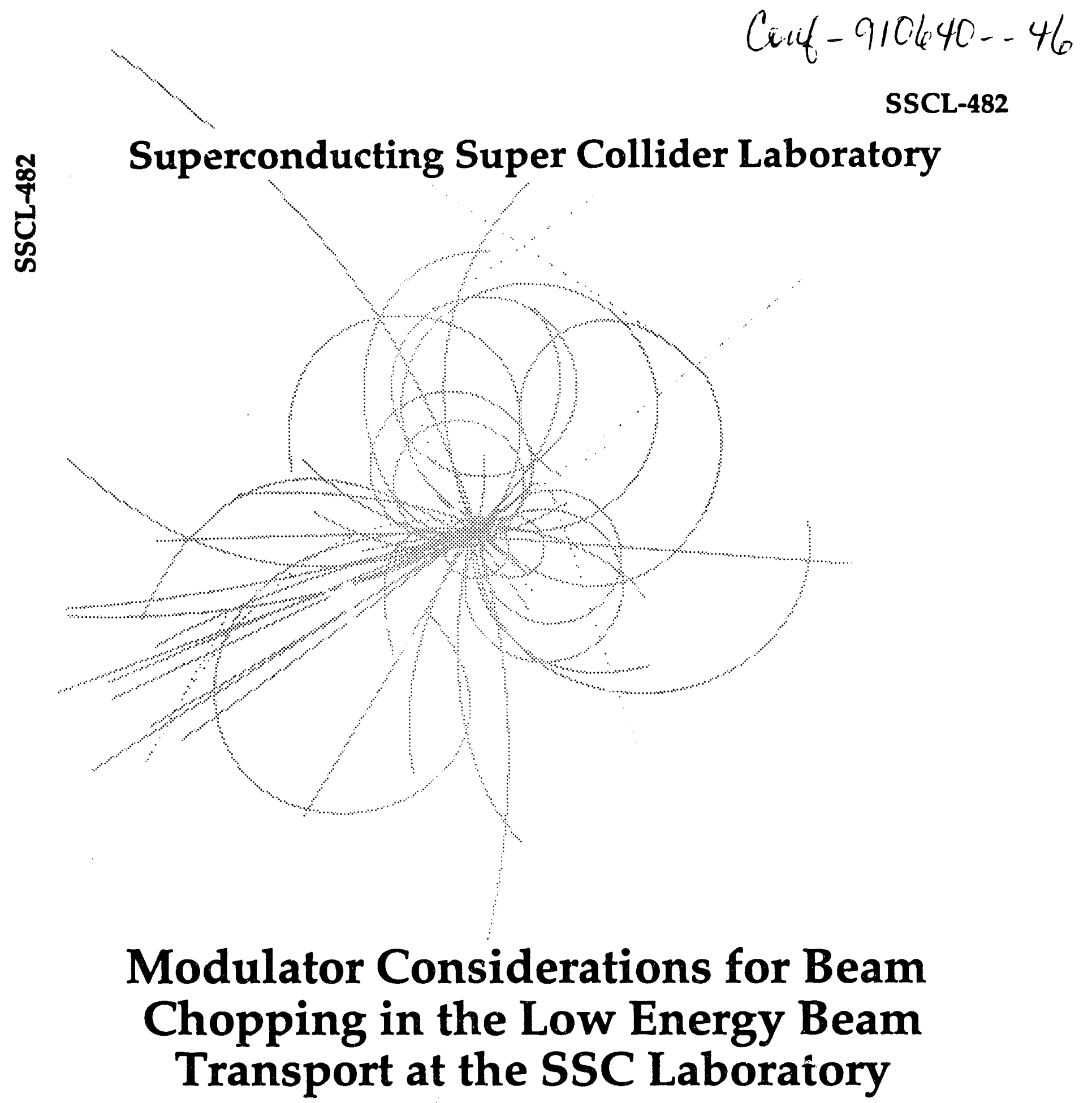

D. Anderson and G. Pappas

June 1991

$$
\text { Ho bu USTl }
$$




\title{
Modulator Considerations for Beam Chopping in the Low Energy Beam Transport at the SSC Laboratory*
}

\author{
D. Anderson and G. Pappas
}

Accelerator Division

Superconducting Super Collider Laboratory ${ }^{\dagger}$

2550 Beckleymeade Ave.

Dallas, TX 75237

June 1991

*Presented at the IEEE 8th Pulsed Power Conference, June 17-19, 1991.

tOperated by the Universities Research Association, Inc., for the U.S. Department of Energy under Contract No. DE-AC02-89ER40486. 
MODOLATOR CONSIDERATIONS FOR BEAY CHOPPING IN THE LON ENERGY BEAM TRANSPORT AT THE SSC IABORATORY*

\author{
D.E. Anderson and G.C. Pappas \\ Accelerator Division \\ Superconducting Super Collider Laboratory \\ Dallas, Texas 75237-3946
}

\section{Abstract}

Beam chopping in the low energy transport line at the Superconducting Super Collider Iaboratory is accomplished using an electrostatic deflection system. IINAC requirements dictate the design of two modulators operating at $10 \mathrm{~Hz}$ with rise and fall times (as measured from approximately 10-998) of $-100 \mathrm{~ns}$. Design of the first pulser, normally at $10 \mathrm{kV}$ and pulsed to ground potential, utilizes a transformer-coupled diode-clamped solid state circuit to achieve the 2-35 $\mathrm{Hs}$ pulse width range required. The second pulser, which pulses from ground to approximately $7 \mathrm{kV}$, relies on a series vacuum tube circuit. The current designs, as well as recent test results and other circuit topologies considered, will be presented.

\section{Introduction}

The magnetron ion source at the SSC Laboratory produces ions by running a glow discharge through hydrogen. Due to the slow establishment of ion density at the source, an ion bunch sharpener must be used downstream to meet the requirements of the radio-frequency quadrupoles in the next section of the IINAC. Prior to entry into the RFQ, an electrostatic focusing system is used to collimate and gate the diverging beam. Two such focusing systems are under consideration, each of which can be pulsed to provide for beam deflection. The first system is an Einzel lens, with a series of biased and unbiased cylindrical electrodes providing the focusing. The second system, a helical electrostatic quadrupole, or HESQ, utilizes a quadrupole configuration twisted in a helix to provide focusing in both planes. Pulsing of one electrode in either configuration provides deflection necessary to divert the beam from the RFQ aperture, thereby gating the proton beam delivered by the IINAC.

\section{Einzed Iens Pulser}

The Einzel lens diverts beam with a voltage of approximately $10 \mathrm{kV}$. Pulsing the electrode to ground potential allows beam to propagate through the RFQ aperture. Acceptance requirements of the RFQ, to maintain an acceptable energy spread within a beam bunch, require the electrode voltage to remain within 1 of the operating voltage during the pulse and within

Foperated by the Oniversities Research Association, Inc., for the O.S. Department of Energy under Contract No. DE-AC02-89ER40486.
10 of a variable 5-10 kV bias voltage when beam is being diverted. A variable pulse width is necessary for the various operating conditions of the Super Collider.

Due to the biased nature of the lens, a transformer-coupled circuit topology was considered. The isolation provided by the transformer allows the secondary to float at $-10 \mathrm{kV}$ dc while diverting beam, and can couple a pulse through when beam is propagating. It was determined that a pulse transformer with $10 \mathrm{kV}$ dc primary to secondary isolation able to pass pulses with rise and fall times less than 100 ns showed appreciable droop, on the order of 208 for the optimum design. Therefore, if such a topology was to be used, a method of compensating for the droop would have to be developed. Another topology was considered, using a series-shunt array of vacuum tubes. The series tube would ramain conducting during the da bias, turning off during the pulse while the shunt tube pulls the capacitive load to ground potential. This system has the advantage of requiring no transformer, but suffers from lifetime and reliability problems associated with gaseous - lectronic devices. Furthermore, tube drop as well as drift between pulses and during the lifetime unst be considered. Since the transformer option uses passive and solid state devices, its lifetime and reliability exceeds that of tha tube option and therefore it is the design of choice.

The modest voltages involved, along with the Low peak currents necessary to charge the small stray capacitive load, allowed for the use of a <1500V primary with a moderate stepup pulse transformar. Osing a $15: 1$ stepup transformer, primary voltages can be kept to $-1000 \mathrm{~V}$ (allowing for switch losses) and peak currents on the primary <100 A. The low primary voltage enabled the use of solid-state switching devices capable of turning on and off on the primary. FETs, Insulated Gate Bipolar Transistors (IGBTs), and Static Induction Transistors (SITs) were all considered for switching in the primary circuit. FETs and IGBTs, with the necessary switching times, had maximum holdoff voltages of $\sim 800$ $V$ and peak pulsed current handling of $\sim 60 \mathrm{~A}$, requiring series-shunt arrays to meet the pulser primary requirements. SITs, on the other hand, can holdoff up to $1500 \mathrm{~V}$ and conduct peak currents up to $-300 \lambda$ in a single device. Furthermore, due to their multichanneling, they displa: Low voltage drops during conduction. With no potential barrier in the channel when zero gate bias is applied, the devices are normally on and therefore can be made to conduct current quickly, particularly when applying a 
positive gate bias. Similar to a vacuum tube, a modest $(-60 \mathrm{~V})$ negative voltage on the base inhibits conduction. The moderate gate capacitance and high input resistance require only moderate current gate drivers. Furthermore, the relatively -inear gate voltage verses drain-tosource current characteristics allow for linear operation in the negative gate voltage region.

Three topologies were considered for compensation of the droop inherent in the fast pulse transformer. Generically, they are shown in Figure 1, with difierent components used in different topologies. The first technique utilizes a capacitive clipping circuit on the primary (consisting of $D_{1}$ and $C_{c l}$ p, without $Q_{2}$ and $\left.D_{2}\right)$, with a capacitor $\left(C_{c l 1 p}\right)$ holding the primary voltage constant during the pulse. The clipping nature of this circuit diverted some of the primary current from the transformer, charging the clipping capacitor, and thereby caysing the load voltage to actually ramp up slfghtly. Furthermore, due to the capacitive lodd, the load voltage actually oscillated significantly through the transformer leakage inductance. Great care was also necessary to insure tnat the ratio between charge voltage and clipping voltage were kept constant, otherwise inadequate or excessive clipping occurred. To avoid these problems, another approach was taken.

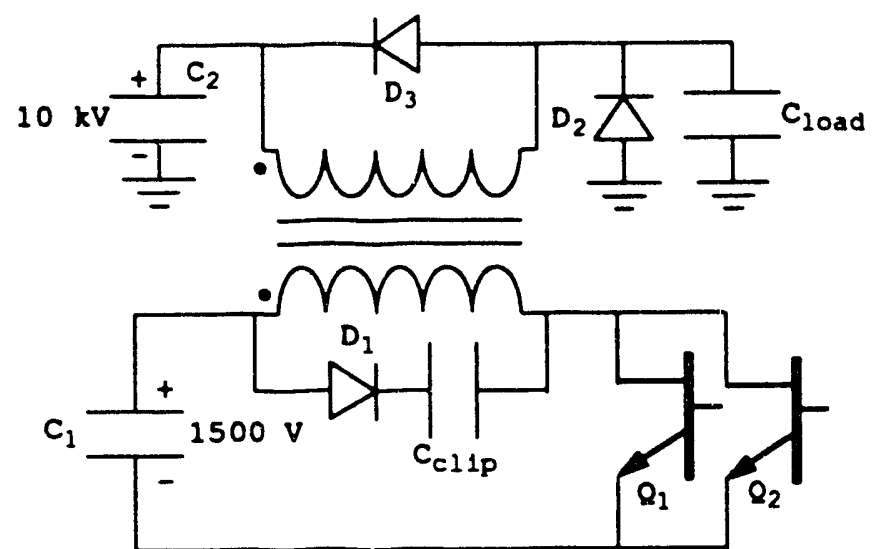

Figure 1. Generic Circuit Schematic Einzel Lens Pulser

A second technique considered utilized two SIT transistors on the primary. The first $\left(Q_{1}\right)$ was driven into saturation and acted as the switch. The second $\left(Q_{2}\right)$, in parallel with the switching SIT, utilized voltage feedback from the load and linearly compensated the droop by supplying excess current to feed the transformer open circuit inductance $\left(D_{1}, D_{2}\right.$, and $C_{c l i p}$ not in circuit). Figure 2 shows uncompensated and compensated waveforms using this technique.The operating voltage of the compensated waveform exceeds that of the uncompensated waveform due to improper feedback amplifier gain. Oscillations on the front end of the pulse are the result of oscillations on the feedback amplifiers driving $Q_{2}$. Droop compensation is fairly good for the first -70 of the pulse flattop (ignoring oscillations), but is unac- ceptable during the last 308. This is primarily due to the fact that in order to cause $Q_{2}$ to conduct large enough currenta to supply the transformer open-circuit inductance at the end of the pulse, the transistor must be driven into saturation. However, with further refinement of the feedback amplifiers to reflect these nonlinearities, or consideration of other topologies, a designer could conceivably build a circuit capable of controlling the pulse flattop to the desired tolerance.

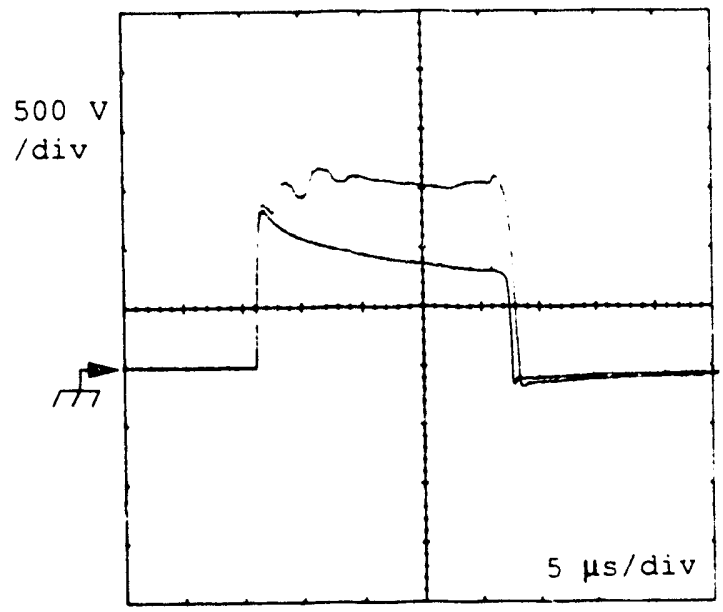

Figure 2. Active Droop Compensation

The third technique uses $D_{2}$ (without $Q_{2}, D_{1}$, or $\left(C_{\text {clip }}\right)$ to clip the secondary voltage to within a diode voltage drop of ground potential. The primary is intentionally charged to a voltage such that the primary voltage multiplied by the turns ratio is about 150 of the operating voltage, thereby insuring the diode $D_{2}$ turns on rapidly. Figure 3 shows this concept. Adjusting this charge voltage (within component limitations) also allows the designer to overcome transformer limitations; i.e. if the transformer is slower than required, overshooting the secondary voltage by 2 or 3 times is analogous to measuring the actual risetime from 0508 or $0-331$, respectively.

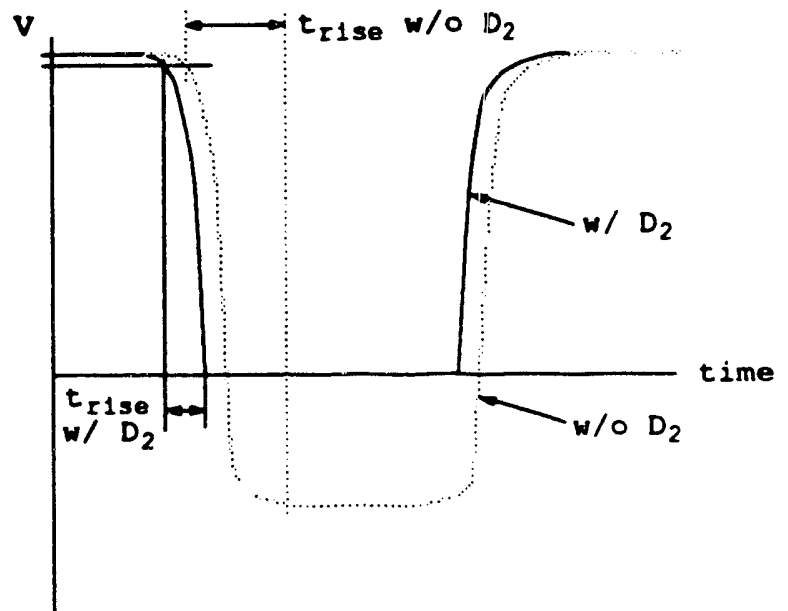

Figure 3. Diode Clipping Concept

This third concept is presently being pursued for this pulser design. Its use of passive 
components, somiconductor switching elements, and no linear active devices makes it an attractive choice. Figure 4 shows actual data taken from a test circuit, showing the effect on the waveform for the circuit. A 5:1 stepup transformer (much faster than the transformer to be used in the actual circuit) is used, with the primary charged to $-300 \mathrm{~V}$. The load capacitance is simulated with a $-10 \mathrm{pF}$ capacitor, and rectifier diodes are used in place of the diodes to be used in the actual circuit. The secondary is biased to $\sim 800 \mathrm{~V}$. As seen, the rise and fall times are on the order of $100 \mathrm{~ns}$, and clipping occurs at about ground potential. Although not shown, the flattop (ignoring under- and overshoot) is within specification, as would not be the case if not for the clamping diode.

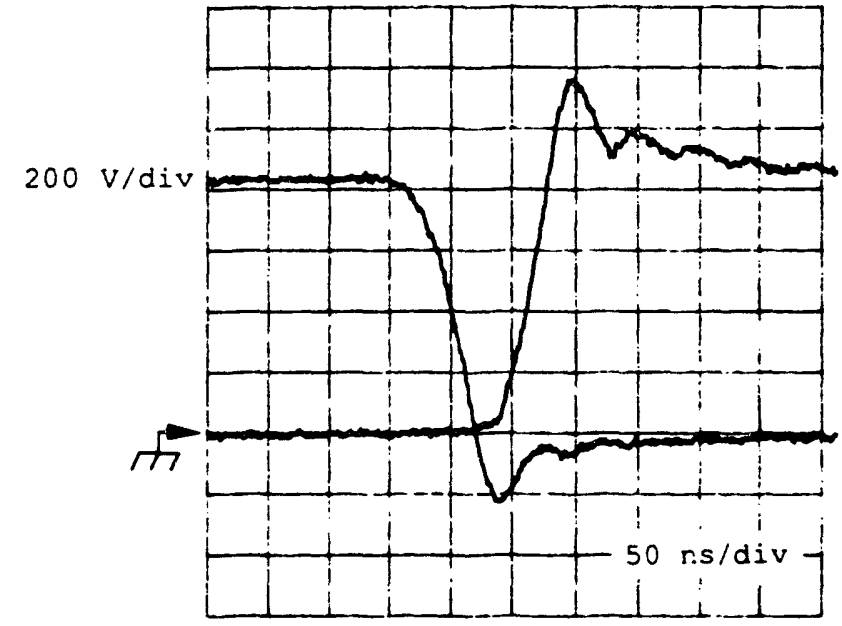

Figure 4. Droop Compensation using Diode Clamping

\section{HESQ PULSER}

Without any yjltage applied to one of the quadrupole electrodes, beam is diverted from the RFQ aperture. Application of a $\rightarrow \mathrm{kV}$ voltage pulse allows beam to propagate through the RFQ. As was the case for the Einzel lens pulser, the pulsed voltage must not vary by more than 1 s during the pulse, and must remain within $1 \mathrm{kV}$ of ground potential when beam is being diverted. A 2-35 $\mu$ s variable pulse width is necessary for running the Super Collider in test beam, commissioning, and collider fill modes.

For this design, it was decided to avoid pulse transformers, since droop compensation could only be achieved using zener c'ode clamping or some other variation on the ther two techniques presented earlier. Zeners are inherently slow devices, and were deemed unacceptable to meet the $100 \mathrm{~ns}$ rise and fall time requirements. Thus, avoiding the transformer required switching to occur at high voltages. Furthermore, the variability of the pulse width eliminates the option of using a pulse-forming network with a closing switch. Therefore, a high voltage opening and closing switch, capable of conducting modest peak currents, was required. Solid state devices could be used, but would reguire large series arrays to hold off the required $\sim 7 \mathrm{kV}$. It was therefore decided to use a planar triode.

The circuit shown in Figure 5 is the design being pursued. A $4 \mu F\left(C_{1}\right)$ capacitor is charged to operating voltage, and then discharged through the planar triode (Eimac Y690). A $1 \mathrm{k} \Omega$ resistor in parallel with the capacitive load is required to discharge the load capacitor at the end of the pulse in $<100 \mathrm{~ns}$. Since the stray capacitance introduced by the isolation transformer must also be pulsed, its value was kept below $20 \mathrm{pF}$ to meet the fall time requirements. Also, the coaxial cable necessary adds to the capacitance of the load, and therefore $75 \Omega$ RG11 cable was chosen to minimize the capacitance.

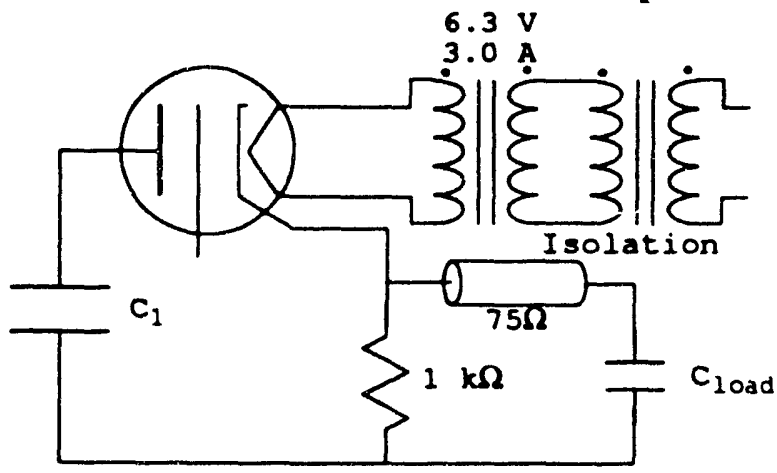

Figure 5. HEso Pulser Circuit

Figure 6 shows the load voltage (l kV/division vertical scale) on the test circuit operating at $5 \mathrm{kV}$. As shown, the rise and fall times do not fall into specification due to the additional capacitance added by the coaxial cable (12" of $50 \Omega$ cable in test circuit). However, due to the strong steering mechanism of the HESQ, only the pulse transition over the last 2 kV is important, and that occurs in approximately $1 \mathrm{CO}$ ns. The fall time is still slow, due to the slow recovery p-channel MOSFETs used to drive the tube in the test circuit. The flattop, although not completely shown, is well within the $50 \mathrm{~V}$ tolerance required.

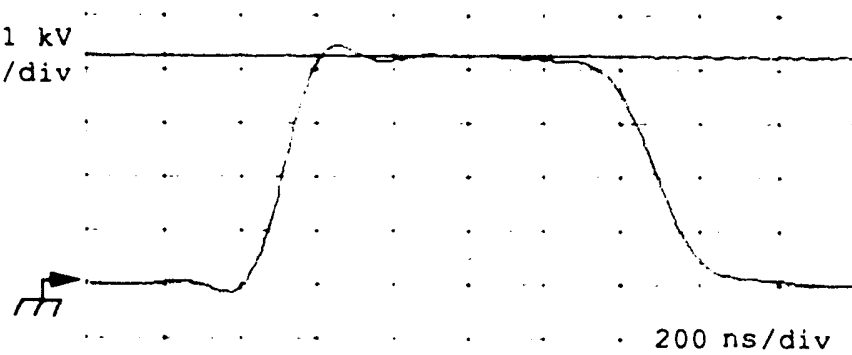

Figure 6. HESQ Pulser Rise and Fall Times

\section{Conclusion}

Beam gating in the low energy beam transport 
stage of the LINAC at the SSC Laboratory is accomplished using a pulsed electrostatic systom. Two systems, to mer the different requirements of the two electrostatic systems, are presently under development. The first pulser, used on the Einzel lens, is pulsed from a $10 \mathrm{kV}$ dc bias to ground potential using a transformercoupled diode-clamped scheme. The other pulser is pulsed from ground potential to $\sim 7 \mathrm{kV}$ for use on the HEso lens, and utilizes a series vacuum tube configuration. Simulations and breadboard circuits have demonstrated the ability of the two circuits to meet the rise and fall time requirements. Limitations of the breadboard circuits in meeting other pulse parameters have been addressed and should be alleviated in the final system. 

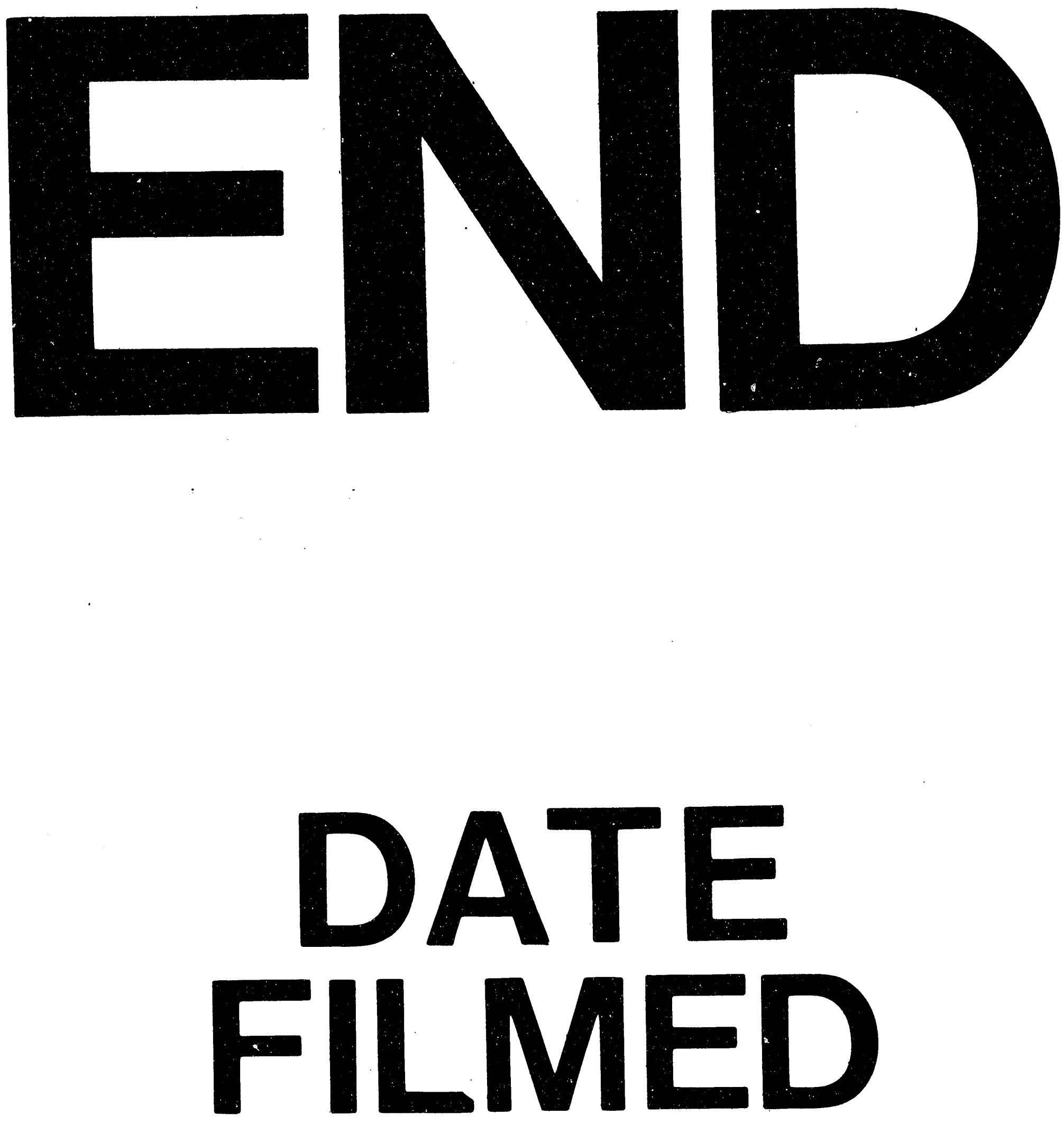

1

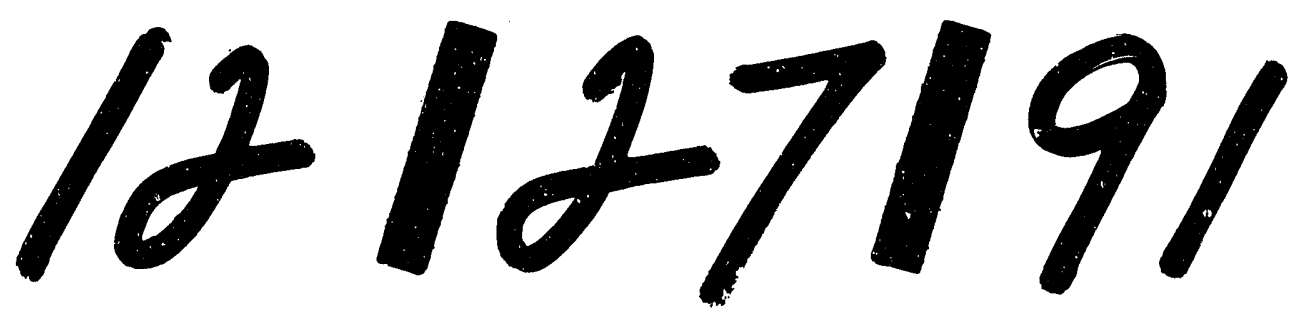

7 
